\title{
Comportamiento sexual de Alpaida veniliae (Araneae: Araneidae)
}

\author{
Marco A. Benamú ${ }^{1}$, Norma E. Sánchez ${ }^{2}$, Carmen Viera ${ }^{1}$ \& Alda González ${ }^{2}$ \\ 1. Laboratorio Ecología del Comportamiento, Instituto de Investigaciones Biológicas Clemente Estable, Av. Italia $\mathrm{N}^{\circ}$ \\ 3318 (11600) Montevideo, Uruguay; marcobenamu@gmail.com, cviera@fcien.edu.uy \\ 2. Centro de Estudios Parasitológicos y de Vectores (CEPAVE) (Centro Científico Tecnológico-CONICET-La Plata) \\ (Universidad Nacional de La Plata). 2 No 584 (1900) La Plata, Argentina; normasanchez3@ciudad.com.ar, \\ asgonzalez@cepave.edu.ar
}

Recibido 30-IX-2011. Corregido 28-II-2012. Aceptado 26-III-2012.

\begin{abstract}
Sexual behavior of Alpaida veniliae (Araneae: Araneidae). Studies in transgenic soybean crops in Buenos Aires province, Argentina, revealed that Alpaida veniliae is one of the most abundant species in the guild of orb web spiders. This species is an effective natural enemy of insect pests affecting this crop. In the present study we carried out a descriptive and quantitative analysis of sexual behavior (courtship, mating and post-mating) of $A$. veniliae. The spiders were collected in transgenic soybean crops located in Chivilcoy $\left(35^{\circ} 01^{\prime} \mathrm{S}-60^{\circ} 06^{\prime} \mathrm{W}\right)$, Buenos Aires, Argentina, and reared under laboratory conditions. Based on observations of 20 couples (with virgin females), behavioral units of male and female in terms of postures and movements, including details on duration and frequency, were described at all stages of sexual activity (courtship, mating and post-mating). Courtship exhibited the greatest number and duration of behavioral units in both sexes. Male and female had a sequence of 16 and nine units, respectively, being the frequency of repetitions of the units significantly higher in the male. Mating was brief and males used a single palp to fill only one of the female spermathecae, after which the female became unreceptive. Mating had two behavioral units in the male and only one in the female. During post-mating males had three and females two behavioral units. The average duration of the whole sexual behavior was $541.90 \pm 123.1$ seconds for the male and $338.20 \pm 74.1$ seconds for the female. Alpaida veniliae females rarely accept a second mating with the same or another male (remating), indicating a strict monogamy. In $46 \%$ of observed mating, the female cannibalized the male after it. Females became unattractive after mating, since stop producing sex pheromones, causing a reduction of the male vibratory courtship. The high cost of courtship, including the risk of cannibalism, would reinforce the selectivity of males towards receptive virgin females. Rev. Biol. Trop. 60 (3): 1259-1270. Epub 2012 September 01.
\end{abstract}

Key words: Araneidae, Alpaida veniliae, orb web spiders, sexual behavior.

El estudio del comportamiento sexual de las arañas tiene importancia debido a que responde a modelos específicos poco variables, éste se considera como un criterio válido para la determinación específica (González \& Armendano 1995). Por otro lado, su estudio junto con el de la genitalia permite conocer aspectos relevantes de su biología reproductiva (Huber 1994, 1995, Uhl et al. 1995), y de la selección sexual (Eberhard 1985, 1996, 2004). El cortejo involucra señales químicas, visuales, táctiles y vibratorias, donde uno o ambos sexos participan activamente. Habitualmente, es el macho el que desarrolla formas diferentes y complejas de acercarse a la hembra para maximizar su éxito reproductivo o supervivencia. El cortejo sirve como un código de reconocimiento que impide, en general, el apareamiento entre especies emparentadas además de ser útil para la evaluación por parte de la hembra en la elección de pareja (Eberhard \& Huber 1998). De acuerdo a Foelix (1996) el cortejo de las arañas involucra semioquímicos y vibraciones.

En las arañas tejedoras, el comportamiento de cortejo está relacionado con las vibraciones sobre la tela realizadas por los machos, 
que pude funcionar como señal de identificación del macho para el reconocimiento de la especie, al inhibir la agresividad y estimular el comportamiento de apareamiento de la hembra (Maklakov et al. 2003). González \& Armendano (1995) también consideran que las señales vibratorias están relacionadas con los rituales de cortejo como una preparación hacia el apareamiento.

En Araneidae, los machos utilizan un solo palpo durante la cópula y llenan sólo una de las espermatecas de la hembra y para utilizar el segundo palpo con la misma hembra, tienen que volver a cortejar (Schneider et al. 2006, Herberstein et al. 2005). En esta familia es característica la presencia de canibalismo y los machos que sobreviven a la primera cópula pueden volver a copular con el otro palpo (Elgar \& Nash 1988, Schneider \& Lubin 1998, Fromhage et al. 2003, Herberstein et al. 2005, Nessler et al. 2007, Uhl et al. 2007).

Estudios realizados en el cultivo de soja transgénica en la provincia de Buenos Aires, Argentina (Benamú 2010), revelaron que la araña Alpaida veniliae es una de las especies más abundantes del gremio de arañas tejedoras orbiculares. Esta especie es numéricamente importante en el cultivo y resulta ser un eficaz enemigo natural de los insectos plaga, debido a sus hábitos predadores y por encontrarse presente, en distintos estados de desarrollo, durante todo el ciclo del cultivo (Benamú 2010, Benamú et al. 2011). Son pocos los estudios relacionados al comportamiento sexual de arañas tejedoras asociadas a cultivos agrícolas en la Argentina. Por lo tanto, para comprender mejor el desempeño de $A$. veniliae en condiciones de campo, es importante conocer los aspectos básicos de su reproducción, lo cual incluye el encuentro sexual, el cortejo y la cópula.

En el presente trabajo se realiza un análisis descriptivo del comportamiento sexual (cortejo, cópula y post-cópula) de Alpaida veniliae.

\section{MATERIALES Y MÉTODOS}

Cría de Alpaida veniliae: Las arañas se recolectaron en cultivos de soja transgénica localizados en Chivilcoy $\left(35^{\circ} 01^{\prime} \mathrm{S}-60^{\circ} 06^{\prime} \mathrm{W}\right)$ (Buenos Aires, Argentina). En el laboratorio se acondicionaron en cajas plásticas de Petri de $6 \mathrm{~cm}$ de diámetro, a $25 \pm 2^{\circ} \mathrm{C}$ de temperatura, $75 \pm 5 \%$ de humedad relativa y un fotoperiodo de $16 \mathrm{~h}$ luz y $8 \mathrm{~h}$ de oscuridad.

Los individuos recolectados en el campo, así como los emergidos de las ootecas construidas en el bioterio, se alimentaron semanalmente con Drosophila melanogaster hasta el sexto estadio (juveniles grandes) y luego con Musca domestica hasta adultos.

Observación y descripción de cortejo y cópula: La observación comenzó con 20 hembras vírgenes adultas colocadas individualmente en bastidores de madera de $15 \mathrm{~cm}$ de alto, $10 \mathrm{~cm}$ de ancho y $5 \mathrm{~cm}$ de espesor, con vidrios corredizos, para la construcción de la tela. Una vez terminada la construcción se alimentaron con Musca domestica en dos etapas: (i) dos días antes de introducir el macho, (ii) dos horas antes de la introducción del mismo, para minimizar el efecto de canibalismo por hambre. Posteriormente en cada bastidor se colocó un macho en la parte inferior del mismo, con el fin de permitirle detectar los hilos de seda de la hembra sobre el sustrato.

Para la descripción de las unidades de comportamiento y la duración de las mismas, durante todas las etapas del apareamiento (cortejo, cópula y post-cópula), se siguió la observación de estas 20 parejas. Todas las hembras fueron expuestas a re-cópula con el mismo macho o con otro macho y la observación se terminó cuando la hembra ahuyentó al macho.

Las cópulas se registraron a través de fotografías y filmaciones digitales y se revisaron cuadro a cuadro (30 fotogramas/segundo) a través del programa Pinnacle Studio 9 (Pinnacle System, Inc. 2003).

Siguiendo a Font et al. (1998) se describieron las unidades de comportamiento del macho y de la hembra en términos de posturas y movimientos, se incluyeron detalles relativos a su duración y frecuencia en todas las etapas del apareamiento. 
Como los datos de duración de las diferentes unidades de comportamiento no tuvieron distribución paramétrica, se utilizó la prueba no paramétrica de Kruskal-Wallis para comparar los valores durante el cortejo, cópula y postcópula, en cada sexo. Además, se estimaron las diferencias de la duración en el cortejo y postcópula entre ambos sexos. Se realizó la comparación de las medianas mediante el método gráfico de Box y Wisker Plot. Para comparar la frecuencia media de repetición de las unidades de comportamiento en ambos sexos, se utilizó la prueba t de Student. En todas las pruebas, $\mathrm{p} \leq 0.05$ fue considerado significativo. Los análisis estadísticos se realizaron utilizando STATGRAPHICS Centurion XV, versión 2.15.05.
(StatPoint, Inc. 2007) y BioEstat 5.0 (Ayres et al. 2007).

\section{RESULTADOS}

Las posiciones adoptadas por ambos sexos durante la totalidad del comportamiento sexual se describen en el cuadro 1.

Las unidades de comportamiento del macho y de la hembra de $A$. veniliae y la duración de las mismas son descritas en los cuadros 2,3 y 4 .

El cortejo constituyó la etapa con el mayor número y duración de unidades del comportamiento sexual de $A$. veniliae. El macho presentó un total de 16 unidades de comportamiento con

CUADRO 1

Posiciones adoptadas durante el comportamiento sexual de Alpaida veniliae

TABLE 1

Positions adopted during the sexual behavior of Alpaida veniliae

\begin{tabular}{|c|c|c|c|}
\hline \multirow{2}{*}{ Posiciones adoptadas } & \multicolumn{2}{|c|}{ Descripción } & \multirow{2}{*}{ Figuras } \\
\hline & Hembra & Macho & \\
\hline $\begin{array}{l}\text { Posición } 1 \\
\text { (Posición de reposo y } \\
\text { de acicalamiento) }\end{array}$ & $\begin{array}{l}\text { Suspendida en el "hub" de la tela (sitio } \\
\text { donde se encuentra en estado de reposo), } \\
\text { verticalmente con el cefalotórax hacia } \\
\text { abajo }\end{array}$ & $\begin{array}{l}\text { Suspendido horizontalmente, con el dorso } \\
\text { hacia abajo y el cefalotórax hacia la } \\
\text { hembra }\end{array}$ & 1 \\
\hline Posición 2 & Igual a posición 1 & $\begin{array}{l}\text { Suspendido, inclinado con el cefalotórax y } \\
\text { dorso hacia arriba y enfrentado a la hembra }\end{array}$ & 2 \\
\hline Posición 3 & $\begin{array}{l}\text { Suspendida, inclinada con el cefalotórax } \\
\text { hacia abajo, dorso hacia arriba y hacia el } \\
\text { macho }\end{array}$ & Igual a posición 2 & 3 \\
\hline Posición 4 & $\begin{array}{l}\text { Suspendida en el "hub" de la tela, } \\
\text { verticalmente con el dorso hacia el macho. }\end{array}$ & $\begin{array}{l}\text { Suspendido, inclinado con el cefalotórax y } \\
\text { dorso hacia abajo, y opuesto a la hembra }\end{array}$ & 4 \\
\hline Posición 5 & $\begin{array}{l}\text { Suspendida, inclinada con el cefalotórax } \\
\text { hacia arriba y con el dorso hacia abajo, } \\
\text { enfrentada con el macho }\end{array}$ & $\begin{array}{l}\text { Suspendido, inclinado con el cefalotórax } \\
\text { y dorso hacia abajo, enfrentando con la } \\
\text { hembra. }\end{array}$ & 5 \\
\hline Posición 6 & $\begin{array}{l}\text { Suspendida, inclinada con el cefalotórax } \\
\text { y dorso hacia abajo, y con la zona ventral } \\
\text { hacia el macho }\end{array}$ & Igual a posición 5 & 6 \\
\hline Posición 7 & $\begin{array}{l}\text { Suspendida verticalmente con el } \\
\text { cefalotórax hacia abajo y con la zona } \\
\text { ventral hacia el macho }\end{array}$ & $\begin{array}{l}\text { Suspendido, inclinado por las patas III } \\
\text { y IV y dorso hacia abajo. Patas I y II } \\
\text { dirigidas hacia la hembra, palpos frotando } \\
\text { el epigino de la hembra }\end{array}$ & 7 \\
\hline $\begin{array}{l}\text { Posición } 8 \\
\text { (Posición de cópula) }\end{array}$ & Igual a posición 7 & $\begin{array}{l}\text { Suspendido del hilo verticalmente, } \\
\text { cefalotórax hacia abajo, palpo hacia el } \\
\text { epigino de la hembra }\end{array}$ & 8 \\
\hline
\end{tabular}


CUADRO 2

Descripción de las unidades del comportamiento sexual del macho de Alpaida veniliae

TABLE 2

Description of units of the male sexual behavior of Alpaida veniliae

Unidades

\section{Cortejo}

Detección

Presencia-I

Construcción hilo de apareamiento

Pedaleo de patas I y II

Presencia-II

Pausa

Movimiento de alejamiento-acercamient

Sacudidas abdominales

Toqueteo de patas

Estimulación palpal

Toma de posición

\section{Cópula}

Abrazo

Inserción palpal

\section{Post-Cópula}

Retirada

Quietud

Limpieza palpal
Reconocimiento de los hilos de la hembra, golpeteo de los palpos y las patas I y II sobre hilos

Movimiento vibratorio con patas III, alternado, sobre el hilo de la tela de la hembra

Hilo para realizar el apareamiento en el borde de la tela de la hembra

Pedaleo con patas I y II sobre el hilo de apareamiento

Movimientos vibratorios con patas III sobre el hilo de apareamiento

Ausencia de todo tipo de movimiento o actividad por no más de 60 segundos, para luego retomar la actividad

Movimientos sobre hilo de apareamiento dirigidos hacia la hembra

Movimientos abdominales dorsoventrales, el impulso inicial es dorsal

Movimiento de patas I y II del macho sobre patas I y II de la hembra

Roce de los palpos alternadamente sobre el epigino de la hembra

Movimientos para adoptar la posición de apareamiento

Abrazo a la hembra por el abdomen inmovilizándola

Inserción palpal en el epigino de la hembra

Macho se retira cortando el hilo de apareamiento después de haber finalizado la cópula

Ausencia de todo tipo de movimiento o actividad, quedando el macho colgado del hilo con todas las patas extendidas en estado cataléptico, por un tiempo mayor a 60 segundos

Limpieza de los palpos frotándolos con las patas I y II
Posiciones

Frecuencia (n repeticiones)

$\begin{array}{cc}\text { Posición } 2 & 1 \\ \text { Posición 2 } & 9 \\ \text { Posición 2 y } 4 & 3 \\ \text { Posición 5 y } 6 & 11 \\ \text { Posición 3, } 4 \text { y } 5 & 2 \\ \text { Posición } 3 \text { y } 4 & 3 \\ \text { Posición } 5 \text { y } 6 & 2 \\ \text { Posición } 6 & 13\end{array}$

Posición 6 y 7

3

Posición 7

Posición 7 y 8

1

Posición 7 y 8

Posición $8 \quad 1$

9 
CUADRO 3

Descripción de las unidades del comportamiento sexual de la hembra de Alpaida veniliae

TABLE 3

Description of the units of female sexual behavior of Alpaida veniliae

\begin{tabular}{|c|c|c|c|}
\hline Unidades & Descripción & Posiciones & $\begin{array}{l}\text { Frecuencia } \\
\text { (n repeticiones) }\end{array}$ \\
\hline \multicolumn{4}{|l|}{ Cortejo } \\
\hline Giro & $\begin{array}{l}\text { Gira en el "hub" con sacudidas en su tela, enfrentando la } \\
\text { zona dorsal hacia el macho }\end{array}$ & Posición 3 & 1 \\
\hline Pausa & $\begin{array}{l}\text { Ausencia de todo tipo de movimiento o actividad por no } \\
\text { más de } 120 \text { s, para luego retomar la actividad }\end{array}$ & $\begin{array}{l}\text { Posición } 1,3 \\
4 \text { y } 6\end{array}$ & 2 \\
\hline Pedaleo de patas I y II & Pedaleo con patas I y II sobre la tela & Posición 2, 6 y 7 & 5 \\
\hline Avance & $\begin{array}{l}\text { Avance hacia la dirección del macho por su tela, hasta tocar } \\
\text { el hilo de apareamiento }\end{array}$ & Posición 5 & 2 \\
\hline Descolgado & $\begin{array}{l}\text { Fijación de un hilo de enganche sobre el hilo de } \\
\text { apareamiento para descolgarse en dirección al macho, } \\
\text { quedando suspendida por las patas III y IV. Patas I y II } \\
\text { plegadas hacia su cuerpo, posición receptiva }\end{array}$ & Posición 7 & 2 \\
\hline Toqueteo de patas & $\begin{array}{l}\text { Movimiento de patas I y II de la hembra sobre patas I y II } \\
\text { del macho }\end{array}$ & Posición 6 & 2 \\
\hline \multicolumn{4}{|l|}{ Cópula } \\
\hline Inserción palpal & Inserción del palpo del macho en el epigino de la hembra & Posición 8 & 1 \\
\hline \multicolumn{4}{|l|}{ Post-Cópula } \\
\hline Retirada & $\begin{array}{l}\text { Regresa al "hub" inmediatamente después de haber } \\
\text { finalizado la cópula }\end{array}$ & Posición 1 & 1 \\
\hline Acicalamiento & $\begin{array}{l}\text { Limpieza del epigino con las patas III de forma alternada, } \\
\text { limpieza de palpos y patas entre sí }\end{array}$ & Posición 1 & 1 \\
\hline
\end{tabular}

una frecuencia media de repetición de $5.54 \pm 4.9$ (media \pm desvío estándar). La hembra exhibió nueve unidades de comportamiento y una frecuencia media de repetición de $2.33 \pm 1.4$. La diferencia en la frecuencia de repeticiones de las unidades de comportamiento entre ambos sexos fue significativa $(t$-test, $\mathrm{t}=1.84, \mathrm{p}=0.04)$.

Los machos exhibieron 11 unidades de comportamiento durante el período de cortejo, representando el $75 \%$ de la duración total del comportamiento sexual, mientras que las hembras efectuaron solo seis unidades, representando el $64.3 \%$ de la duración total. Se observaron diferencias significativas en la duración de las distintas unidades de comportamiento tanto para los machos $(\mathrm{H}=157.32, \mathrm{p}<0.005)$ como para las hembras $(H=97.1, p<0.005)$. Durante la cópula, los machos realizaron dos unidades de comportamiento y las hembras sólo una, mientras que en la post-cópula los machos presentaron tres unidades de comportamiento y las hembras dos, mostrando diferencias significativas entre ellas (machos: $\mathrm{H}=40.81, \mathrm{p}<0.005$; hembras: $\mathrm{H}=29.89, \mathrm{p}<0.005$ ).

La duración total del comportamiento sexual fue significativamente mayor en el macho que en la hembra $(H=22.03, p<0.005)$, determinado por la duración del cortejo $(\mathrm{H}=267.51, \mathrm{p}<0.05)$ y la post-cópula $(\mathrm{H}=83.11$, $\mathrm{p}<0.005$ ).

La hembra de $A$. veniliae siempre fue receptiva durante el cortejo del macho, y 
CUADRO 4

Duración de las unidades del comportamiento sexual del macho y de la hembra de Alpaida veniliae

TABLE 4

Duration of units of sexual behavior of male and female of Alpaida veniliae

\begin{tabular}{|c|c|c|}
\hline \multirow{2}{*}{ Unidades } & \multicolumn{2}{|c|}{ Tiempo (s) (media $\pm \mathrm{DE})$} \\
\hline & Macho & Hembra \\
\hline \multicolumn{3}{|l|}{ Cortejo } \\
\hline Detección & $10.35 \pm 5.6$ (a) & - \\
\hline Presencia-I & $62.85 \pm 47.0(\mathrm{~d})$ & - \\
\hline Giro & - & $3.10 \pm 1.4$ (a) \\
\hline Construcción hilo de apareamiento & $43.25 \pm 56.5(\mathrm{~cd})$ & - \\
\hline Pausa & - & $60.20 \pm 35.4(b)$ \\
\hline Pedaleo patas I y II & $15.95 \pm 17.2(\mathrm{ab})$ & $13.50 \pm 2.6(a)$ \\
\hline Presencia-II & $11.50 \pm 15.8$ (a) & - \\
\hline Avance & - & $5.70 \pm 4.8$ (a) \\
\hline Descolgado & - & $2.75 \pm 0.9$ (a) \\
\hline Pausa & $58.10 \pm 3.9(\mathrm{~d})$ & - \\
\hline Movimiento acercamiento-alejamiento & $13.6 \pm 6.1(\mathrm{ab})$ & - \\
\hline Sacudidas abdominales & $34.95 \pm 38.9(\mathrm{bc})$ & - \\
\hline Toqueteo de patas & $2.20 \pm 0.4(\mathrm{a})$ & $17.5 \pm 8.8$ (a) \\
\hline Estimulación palpal & $55.10 \pm 30.4(\mathrm{~cd})$ & - \\
\hline Toma de posición de cópula & $1 \pm 0$ (a) & - \\
\hline Duración & $308.90 \pm 85.6$ & $102.75 \pm 36.4$ \\
\hline \multicolumn{3}{|l|}{ Cópula } \\
\hline Abrazo e inserción palpal & $10.38 \pm 2.7$ (a) & $10.38 \pm 2.7$ (a) \\
\hline Duración & $10.38 \pm 2.7$ & $10.38 \pm 2.7$ \\
\hline \multicolumn{3}{|l|}{ Post-Cópula } \\
\hline Retirada & $1.20 \pm 0.4$ (a) & $3.45 \pm 2.9$ (a) \\
\hline Acicalamiento & - & $221.60 \pm 70.9(\mathrm{c})$ \\
\hline Quietud & $120.85 \pm 72.7(\mathrm{e})$ & - \\
\hline Limpieza palpal & $100.55 \pm 83.4(\mathrm{e})$ & - \\
\hline Duración & $222.60 \pm 119.7$ & $255.05 \pm 70.3$ \\
\hline Tiempo total trascurrido & $541.90 \pm 123.1$ & $338.20 \pm 74.1$ \\
\hline
\end{tabular}

Letras minúsculas indican diferencias significativas.

Different small letters indicate significant differences.

copuló una sola vez por cada cópula obtenida $(n=20)$, excepto en un único caso donde se observó re-cópula con el mismo macho. Los machos pudieron copular con diferentes hembras (2.05 \pm 1.3$)$. Una vez finalizada la cópula la hembra dejó de ser receptiva y, en algunos casos ahuyentó al macho con movimientos agresivos de ataque $(60 \%$ de los casos) y en otros comió su propia tela $(40 \%)$, impidiendo el comienzo de un nuevo cortejo. Se observó canibalismo de la hembra hacia el macho en el $46 \%$ de las cópulas realizadas.

\section{Sucesión de las unidades del comportamiento sexual del macho y la hembra}

Cortejo: Al ser colocado el macho en el bastidor de la hembra con su tela (posición 1, Fig. 1), inmediatamente realiza una inspección del lugar, encontrando los hilos de la hembra, 
unidad Detección (Fig. 1). Sube por uno de los hilos que conforma el marco de la tela de la hembra realizando las unidades Presencia-I y Desplazamiento (Fig. 2). La hembra gira en el centro de la tela o "hub" (unidad Giro, Fig. 3) y realiza la unidad Pedaleo de patas I y II (Fig. $2,6,7)$, luego se queda quieta por un breve momento (Pausa). El macho construye un hilo de apareamiento desde el borde de la tela de la hembra (Construcción de hilo de apareamiento, Fig. 2, 4), hasta el marco del bastidor. Luego realiza la unidad Presencia-II (Fig. 3, 4, 5) y luego Pausa. Mientras que la hembra está en Pausa, el macho comienza con el Pedaleo de patas I y II (Fig. 5, 6) y Sacudidas abdominales (Fig. 6). La hembra avanza sobre el hilo de apareamiento (Avance, Fig. 5) y nuevamente adopta la unidad Pausa, mientras el macho realiza la unidad Movimiento de alejamientoacercamiento (Fig. 5, 6). La hembra avanza y se descuelga del hilo quedando sujetada sólo con las patas III y IV (Descolgado, Fig. 7), colocándose en la postura de receptividad, posteriormente ambos empiezan con Toqueteo de patas (Fig. 6,7). Finalmente el macho realiza Sacudidas abdominales (Fig. 6) y Estimulación palpal (Fig. 7), concretándose la Toma de posición de cópula (Fig. 7, 8).

Cópula: El macho una vez que logró enganchar el esclerito retrolateral del cymbium con el epigino de la hembra, realiza la unidad Abrazo e Inserción palpal (Fig. 9).

Post-cópula: Una vez terminada la cópula, tras la inserción de un solo palpo, ambos individuos se desenganchan y el macho rápidamente corta el hilo y realiza la unidad de comportamiento Retirada. Simultáneamente, también la hembra realiza la unidad Retirada.
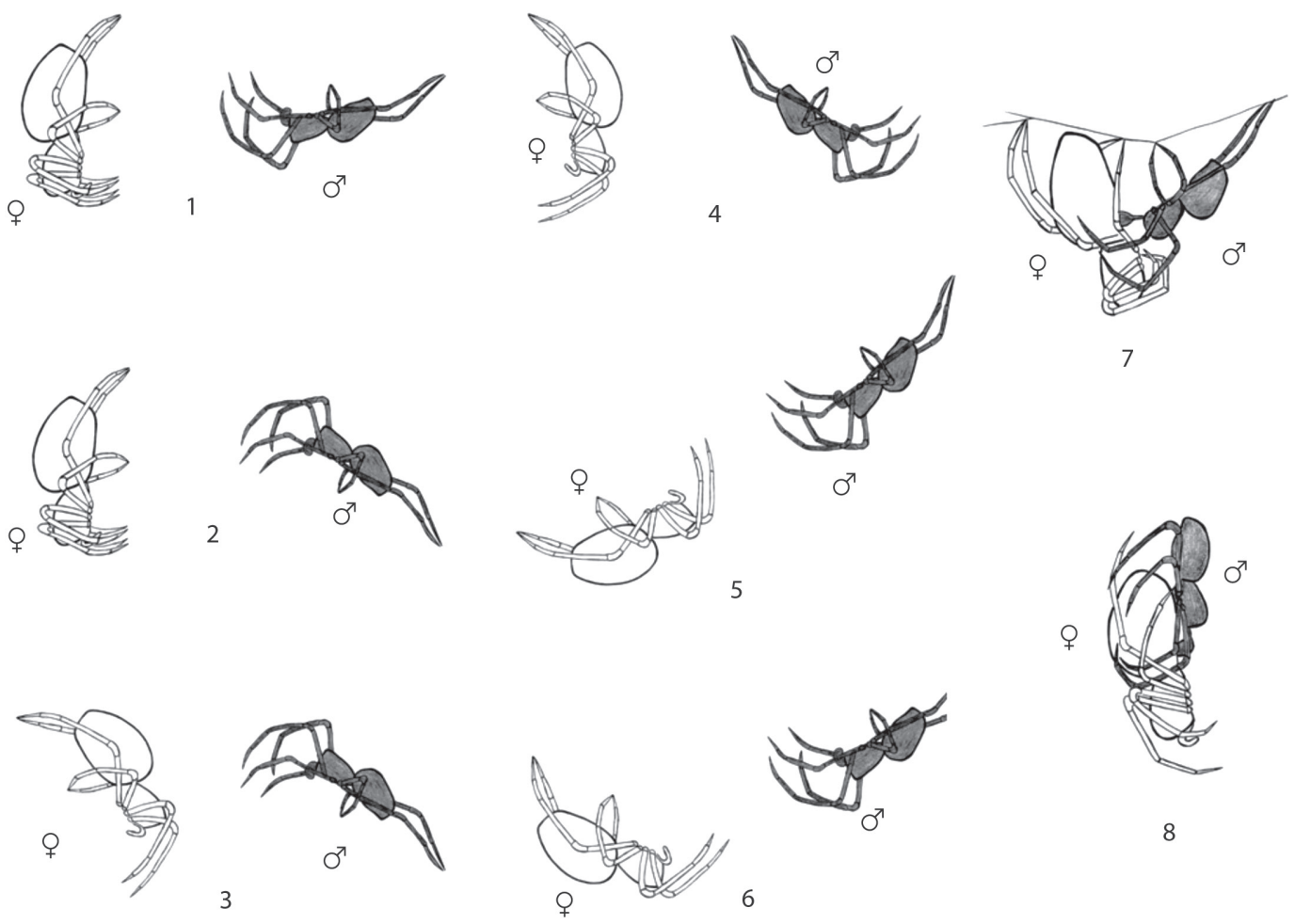

Fig. 1-8. Posiciones adoptadas durante el comportamiento sexual de Alpaida veniliae (hembra en blanco y macho en gris oscuro).

Fig. 1-8. Positions adopted during the sexual behavior of Alpaida veniliae (female in white and male in dark grey). 


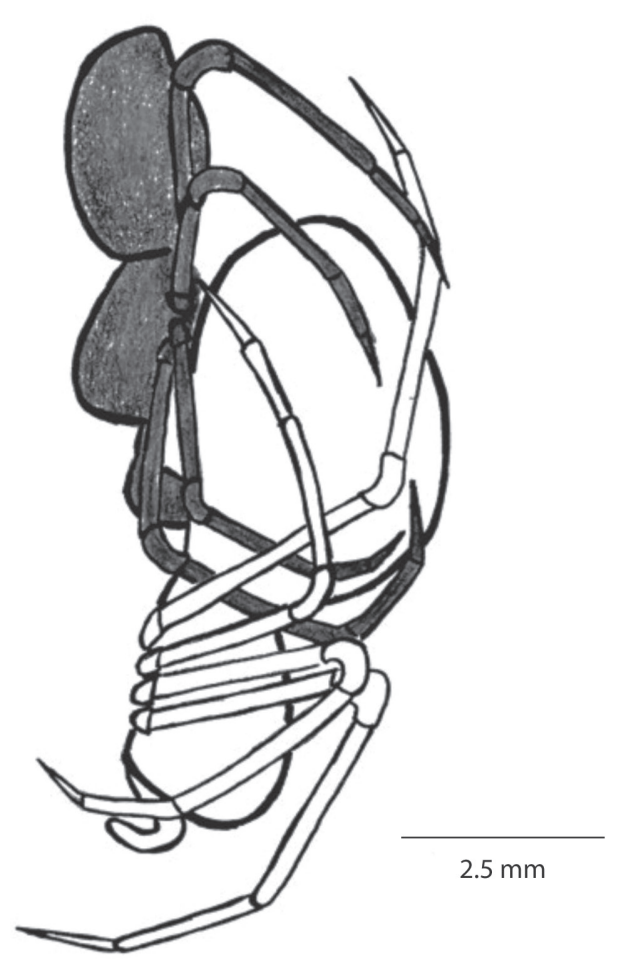

Fig. 9. Posición de cópula "Abrazo" e "Inserción palpal" de Alpaida veniliae (hembra en blanco y macho en gris oscuro).

Fig. 9. Position of couple "Embrace" and "Insertion palpal" of Alpaida veniliae (female in white and male in dark gray).

El macho queda suspendido de su hilo inmóvil cumpliendo la unidad Quietud (Fig. 1), mientras que la hembra comienza con la unidad Acicalamiento en el "hub" (Fig. 1). Posteriormente el macho sube por el hilo y realiza la unidad Limpieza palpal en la parte externa de la tela de la hembra.

\section{DISCUSIÓN}

El comportamiento de cortejo de A. veniliae involucró un mayor tiempo y número de unidades en ambos sexos con relación al comportamiento copulatorio y postcopulatorio, siendo significativamente mayor en el macho. Esto coincide con lo observado por Gerhardt (1924) en Araneus diadematus (Araneidae), por Ross \& Smith (1979) en Latrodectus hesperus (Theridiidae), por González \& Poncio (1992) en Achaearaneae jequitituba (Theridiidae), por Gónzalez \& Armendano (1995) en Achaearaneaea tepidariorum (Theridiidae), y por Eberhard \& Huber (1998) en Leucauge mariana (Tetragnathidae). Este comportamiento involucraría un mayor costo de energía y riesgo de depredación hacia el macho, como lo indican Schneider \& Lubin (1998), congruente con las posibilidades de ser canibalizados o lesionados por las hembras de A. veniliae.

Durante el cortejo de las arañas están involucradas señales químicas, visuales, táctiles y sonoras (Guzmán-Mendoza 2002). Las feromonas femeninas posibilitan a los machos detectar a las hembras sexualmente receptivas e iniciar el cortejo (Roland 1984, Guzmán-Mendoza 2002, Gaskett 2007). El comportamiento vibratorio del macho de $A$. veniliae durante el inicio del cortejo sobre la tela de la hembra, podría estar desencadenado por un estímulo de feromona sexual femenina. En los machos, los quimiorreceptores probablemente estén ubicados sobre los palpos, o en las patas delanteras (Gaskett 2007). Según lo observado en $A$. veniliae, los machos detectarían feromonas de contacto sobre los hilos de la tela de la hembra con los palpos y el primer par de patas (Fraser 1987) antes de iniciar el cortejo.

En las arañas tejedoras el macho presenta secuencias de exhibición y de estimulación mediante vibraciones, en donde la hembra puede responder mostrando receptividad (González \& Poncio 1992). González \& Armendano (1995) sostienen que el comportamiento de cortejo suprime o aminora la tendencia predadora de la hembra, y estimula a ambos sexos para copular. En A. veniliae, este comportamiento fue observado exclusivamente en hembras vírgenes, ya que una vez copuladas excepcionalmente (sólo un caso) aceptaron un segundo encuentro con el mismo macho (recópula), y rechazaron a otros machos, lo cual indica una monogamia estricta.

Durante el inicio del cortejo en $A$. veniliae, el macho realizó vibraciones, permaneciendo inicialmente en el borde del tejido de 
la hembra. De acuerdo a Mercier (2003) los machos pueden estimular a las hembras tironeando los hilos y tamborileando sus palpos en las redes. Casos similares fueron encontrados por Eberhard \& Huber (1998) y Aisenberg \& Eberhard (2009) en Leucauge mariana (Tetragnathidae), en donde el macho tironea de la tela de la hembra con sus patas delanteras, ubicado en la parte externa de la tela. Dichas vibraciones son importantes, ya que reducen el comportamiento agresivo de la hembra, permitiendo al macho evitar ser confundido con una presa (Foelix 1996). Las sacudidas abdominales y los movimientos vibratorios con las patas III realizados por el macho de $A$. veniliae durante el cortejo es coincidente con lo observado por Eberhard \& Huber (1998) en Leucauge mariana (Tetragnathidae). Este mismo comportamiento fue observado en Araneus diadematus (Araneidae) por Gerhardt (1924) y Elgar \& Nash (1988), en Latrodectus hesperus (Theridiidae) por Ross \& Smith (1979), en Dictyna volucripes (Dictynidae) por Starr (1988), en Achaearaneae jequirituba (Theridiidae) por González \& Poncio (1992), en Achaearaneae tepidarorium (Theridiidae) por González \& Armendano (1995) y en Pholcus phalangioides (Pholcidae) por Bartos (1998), y podría ser utilizado con el mismo fin.

La construcción de un hilo de apareamiento por parte del macho de $A$. veniliae, en el borde de la tela de la hembra, es común en arañas tejedoras orbiculares de la familia Araneidae (Guzmán-Mendoza 2002). Este hilo también es utilizado por los machos para cortejar a las hembras por medio de señales vibratorias. Para Guzmán-Mendoza (2002) existe una relación entre los machos que tejen un "hilo de apareamiento" y el tamaño, siendo los machos grandes los que construyen el hilo de apareamiento. Dicho hilo puede estar unido a la red de la hembra de dos formas: (1) al centro como en Argiope aemula (Sasaki \& Iwahashi 1995), y (2) en los límites como en Araneus diadematus (Elgar \& Nash 1988). En este mismo sentido, los machos que utilizan el hilo atado al centro de la red son más pequeños que los que atan sus hilos en los límites. El tamaño "grande" de los machos de A. veniliae (Benamú et al. 2011) con relación a otros machos de Araneidae, y la colocación de "hilos de apareamiento" en los bordes de la tela de la hembra, coincide con lo descrito por Guzmán-Mendoza (2002). El hilo de apareamiento ha sido observado en otras especies por Sasaki \& Iwahashi (1995) en Argiope aemula, Elgar \& Nash (1988) en Araneus diadematus, Herberstein et al. (2002) en Argiope keyserlingi y Schneider et al. (2006) en Argiope bruennichi. Robinson \& Robinson (1978), al analizar la evolución del sistema de cortejo en arañas tropicales, observaron que machos de los géneros Gea, Gasteracantha, Isoxya, Aetrocantha, Micrathena, Cyclosa, Zilla y Eriophora, presentan cortejos basados enteramente en vibraciones sobre el hilo de apareamiento. Estas vibraciones se generan mediante movimientos complejos de patas y cuerpo, y la respuesta de la hembra incrementa estos movimientos, sugiriendo que es un cortejo más avanzado desde el punto de vista evolutivo, coincidiendo con lo observado por nosotros en A. veniliae. Según Herberstein et al. (2002) los machos de Argiope keyserlingi colocan hilos de apareamiento por fuera de la tela de la hembra (como se observó en Alpaida veniliae), para reducir el potencial de lesiones o canibalismo sobre los machos, mientras que a la hembra le permite reducir el costo de pérdida de forrajeo en una red dañada.

Las unidades de comportamiento: Construcción hilo de apareamiento, Presencia I y II, Pedaleo de patas I y II, Sacudidas abdominales, Movimientos de acercamiento-alejamiento, Toqueteo de patas, Estimulación palpal, Toma de posición y Abrazo e Inserción palpal de $A$. veniliae son similares a las descritas por Gerhardt (1924) y por Elgar \& Nash (1988) para Araneus diadematus y algunas de ellas fueron reportadas por Eberhard \& Huber (1998) y Aisenberg \& Eberhard (2009) para Leucauge mariana.

La unidad Toqueteo de patas I y II en machos y hembras de $A$. veniliae durante el cortejo, fue observada también en Leucauge mariana (Eberhard \& Huber 1998, Aisenberg \& Eberhard 2009) y en Glenognatha emertoni 
(Danielson-François 2006). De acuerdo a Eberhard \& Huber (1998) este comportamiento podría constituir una búsqueda o estímulo sensorial en el cortejo del macho hacia la hembra antes de asumir la postura receptiva y posterior cópula.

Debido a la inserción de un solo palpo por parte del macho, la cópula en $A$. veniliae representó solo el $2 \%$ del total de unidades de todo el comportamiento sexual. Esto podría ser suficiente para inseminar a la hembra, tal como ocurre en Argiope bruennichi donde los machos transfieren el $50 \%$ de su carga de esperma (un solo palpo) durante los 10 segundos que dura la cópula, cantidad que resulta suficiente para garantizar la fertilización (Schneider et al. 2005, 2006). En A. veniliae se registró un solo caso donde la duración de la primera cópula fue muy corta y se observó que el macho volvió a cortejar a la misma hembra, ocurriendo la única recópula obtenida, que fue más prolongada que la primera cópula. Este resultado coincide con lo encontrado por Fromhage \& Schneider (2005) y Schneider et al. (2006) en Argiope bruennichi, donde la duración de la cópula estaría directamente relacionada con la cantidad de esperma transferida. La monandría de $A$. veniliae difiere de la hembra de otra especie del mismo género, Alpaida variabilis, que puede copular con varios machos en un mismo periodo de apareamiento (Flórez et al. 2002).

Luego de la cópula, el macho de $A$. veniliae se aleja rápidamente dando un salto y quedando colgado del hilo de apareamiento, coincidiendo con lo observado en Argiope aemula por Sasaki \& Iwahashi (1995). Después de la cópula de $A$. veniliae no hubo cortejo, salvo en el caso singular de la recópula. El esfuerzo que conlleva el intento de recópula con el inicio de todas las etapas desde el cortejo en $A$. veniliae, coincide con lo observado por Sasaki \& Iwahashi (1995) en Argiope aemula y por Schneider et al. (2006) en Argiope bruennichi. De acuerdo a Maklaklov et al. (2003) las hembras se vuelven no atractivas después de la cópula, debido a que dejan de producir feromonas sexuales, provocando una reducción del cortejo vibratorio del macho. Seguramente los costos de cortejo y los obstáculos que los machos tienen que sortear, incluyendo el riesgo de canibalismo, apoyarían la selectividad de los machos hacia hembras vírgenes receptivas.

\section{AGRADECIMIENTOS}

Se agradece a Rodolfo Sosa y Ariel Cabrera por su valiosa ayuda en el campo y laboratorio, a Gabriel Baloriani por los dibujos realizados. Esta investigación fue financiada por la Agencia Nacional de Promoción Científica y Tecnológica de Argentina (ANPCyT).

\section{RESUMEN}

Estudios realizados en cultivos de soja transgénica en la provincia de Buenos Aires, Argentina, revelaron que Alpaida veniliae es una de las especies más abundantes dentro del gremio de arañas orbiculares. Esta especie es un eficaz enemigo natural de las plagas de insectos de este cultivo. En el presente estudio se llevó a cabo un análisis descriptivo y cuantitativo de la conducta sexual (cortejo, cópula y post-cópula) de $A$. veniliae. Las arañas fueron recolectadas en cultivos de soja transgénica ubicados en

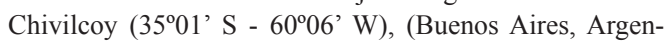
tina) y criadas en condiciones de laboratorio. A partir de la formación de 20 parejas (con hembras vírgenes), se observaron las unidades de comportamiento de machos y hembras, en términos de posturas y movimientos, incluyendo los detalles de su duración y frecuencia en todas las etapas del apareamiento. El cortejo exhibió el mayor número y la mayor duración de las unidades de comportamiento en ambos sexos. La secuencia de unidades fue 16 en el macho y nueve en la hembra, siendo la frecuencia de repeticiones de las unidades significativamente mayor en el macho. La cópula fue breve y el macho usó un solo palpo transfiriendo esperma a una sola espermateca de la hembra. Hubo dos unidades de comportamiento en el macho y una en la hembra. Durante la post-cópula el macho tuvo tres y la hembra dos unidades de comportamiento. La duración media de todo el comportamiento sexual fue $541.90 \pm 123.1$ segundos para el macho y $338.20 \pm 74.1$ segundos para la hembra. La hembra de $A$. veniliae sólo excepcionalmente aceptó una segunda cópula con el mismo u otro macho, lo que indica una monogamia estricta. En el 46\% de las cópulas observadas la hembra canibalizó al macho al terminar la misma. Debido a que a partir de este momento dejan de producir feromonas sexuales, se vuelven no receptivas y provocan una reducción del cortejo del macho. El alto costo del cortejo del macho, incluyendo el riesgo de canibalismo, reforzaría la selectividad de éstos hacia las hembras receptivas vírgenes. 
Palabras clave: Araneidae, Alpaida veniliae, arañas orbiculares, comportamiento sexual.

\section{REFERENCIAS}

Aisenberg, A. \& W. Eberhard. 2009. Female cooperation in plug formation in a spider: effects of male copulatory courtship. Behav. Ecol. 20: 1236-1241.

Ayres, M., M. Ayres Jr., D. Ayres \& A. Santos. 2007. BioEstat 5.0 aplicações estatísticas nas áreas das ciências bio-médicas. Belém, Pará, Brasil.

Bartos, M. 1998. Quantitative analyses of male courtship behaviour in Pholcus phalangioides (Fuesslin, 1775) (Araneae, Pholcidae), p. 171-176. In P.A. Selden (ed.). Proceedings of the $17^{\text {th }}$ European Colloquium of Arachnology, Edinburgh 1997. British Arachnological Society, Burnham Beeches.

Benamú, M. 2010. Composición y estructura de la comunidad de arañas en el sistema de cultivo de soja transgénica. Tesis de Doctorado, Facultad de Ciencias Naturales y Museo, Universidad Nacional de La Plata, Argentina.

Benamú, M., N. Sánchez \& A. González. 2011. Postembryonic development and population parameters of Alpaida veniliae (Araneae, Araneidae), reared in the laboratory. J. Nat. Hist. 45: 1607-1617.

Danielson-François, A. 2006. Natural history of Glenognatha emertoni (Araneae, Tetragnathidae): mating behavior and sperm release in a haplogyne. J. Arachnol. 34: 387-398.

Eberhard, W. 1985. Sexual Selection and Animal Genitalia. Harvard University, Cambridge, Massachusetts, USA.

Eberhard, W. 1996. Female Control: Sexual Selection by Cryptic Female Choice. Princeton University, New Jersey, USA.

Eberhard, W. \& B. Huber. 1998. Courtship, copulation, and sperm transfer in Leucauge mariana (Araneae, Tetragnathidae) with implications for higher classification. J. Arachnol. 26: 342-368.

Eberhard, W. 2004. Male-female conflict and genitalia: failure to confirm predictions in insects and spiders. Biol. Rev. 79: 121-186.

Elgar, M. \& D. Nash. 1988. Sexual cannibalism in the garden spider Araneus diadematus. Anim Behav. 36: 1511-1517.
Flórez, E., J. Pinzon \& A. Sabogal. 2002. Ciclo de vida y parámetros reproductivos de la araña orbitelar Alpaida variabilis (Araneae: Araneidae). Rev. Colomb. Entomol. 28: 183-189.

Foelix, R. 1996. Biology of spiders. Oxford University, New York, Oxford., New York, USA.

Font, E., F. Colmenares \& F. Guillén-Salazar. 1998. El lugar de la etología en las ciencias del comportamiento: un debate inacabado. Rev. Psicol. Gral. Aplic. 51: 55-83.

Fraser, J. 1987. Courtship and copulatory behavior of the funnel-web spider, Hololena adnexa (Araneae, Agelenidae). J. Arachnol. 15: 257-262.

Fromhage, L., G. Uhl \& J. Schneider. 2003. Fitness consequences of sexual cannibalism in female Argiope bruennichi. Behav. Ecol. Sociobiol. 55: 60-64.

Fromhage, L. \& J. Schneider. 2005. No discrimination against previous mates in a sexually cannibalistic spider. Naturwissenschaften 00: 1-4.

Gaskett, A. 2007. Spider sex pheromones: emission, reception, structures, and functions. Biol. Rev. 82: 27-48.

Gerhardt, U. 1924. Weitere Studien über die Biologie der Spinnen. Arch. Naturgesh. 89: 1-225.

González, A. \& G. Poncio. 1992. Análisis del comportamiento sexual de Achaearanea jequirituba Levi, 1963 (Araneae, Theridiidae). Graellsia 48: 9-18.

González, A. \& A. Armendano. 1995. Comportamiento sexual y producción de ootecas por Achaearanea tepidarorium (C.L.Koch) (Araneae, Theridiidae). Rev. Bras. Entomol. 39: 355-369.

Guzmán-Mendoza, R. 2002. Arañas: Reproductoras voraces. Contacto S. 46: 31-41. http://www.izt.uam.mx/ contactos/n46ne/aranas.pdf

Huber, B. 1994. Genital morphology, copulatory mechanism and reproductive biology in Psilochorus simoni (Berland, 1911) (Pholcidae; Araneae). Netherl. J. Zool. 44: 85-99.

Huber, B. 1995. Copulatory mechanism in Holocnemus pluchei and Pholcus opilionoides, with notes on male cheliceral apophyses and stridulatory organs in Pholcidae (Araneae). Acta Zool. Stockholm 76: 291-300.

Herberstein, M., J. Schneider \& M. Elgar. 2002. Costs of courtship and mating in a sexually cannibalistic orb-web spider: female mating strategies and their consequences for males. Behav. Ecol. Sociobiol. $51: 440-446$. 
Herberstein, M., A. Gaskett, N. Vella \& M. Elgar. 2005. Limits to male copulation frequency: sexual cannibalism and sterility in St Andrew's Cross Spiders (Araneae, Araneidae). Ethology 111: 1050-1061.

Maklakov, A., T. Bilde. \& Y. Lubin. 2003. Vibratory courtship in a web-building spider: signalling quality or stimulating the female?. Anim. Behav. 66: 623-630.

Mercier, C. 2003. Vibration transmission characteristics of silk produced by the British cribellate spider Amaurobius similis. University of Leeds, Leeds, West Yorkshire, England. (Consultado: junio 2009, http://www.biolog-e.leeds.ac.uk/Biolog-e/uploads/ clairemercier.pdf).

Nessler, S., G. Uhl \& J. Schneider. 2007. Genital damage in the orb-web spider Argiope bruennichi (Araneae: Araneidae) increases paternity success. Behav. Ecol. 18:174-181.

Pinnacle System, Inc. 2003. Pinnacle Studio 9. Mountain View, California, USA.

Robinson, M. \& B. Robinson. 1978. The evolution of courtship systems in tropical araneid spiders. Symp. Zool. Soc. Lond. 42: 17-29.

Roland, C. 1984. Chemical signals bound to the silk in spider communication (Arachnida, Araneae). J. Arachnol. 11: 309-314.

Ross, K. \& R. Smith. 1979. Aspects of the courtship behavior of the black widow spider, Latrodectus hesperus
(Araneae: Theridiidae), with evidence for the existence of contact sex pheromone. J. Arachnol. 7:69-77.

Sasaki, T. \& O. Iwahashi. 1995. Sexual cannibalism in an orb-weaving spider Argiope aemula. Anim. Behav. 49: 1119-1121.

Schneider, J. \& Y. Lubin. 1998. Intersexual conflict in spiders. Oikos 83: 496-506.

Schneider, J., L. Fromhage \& G. Uhl. 2005. Extremely short copulations do not affect hatching success in Argiope bruennichi (Araneae, Araneidae). J. Arachnol. 33:663-669.

Schneider, J., S. Gilberg, L. Fromhage \& G. Uhl. 2006. Sexual conflict over copulation duration in a cannibalistic spider. Anim. Behav. 71: 781-788.

Starr, C. 1988. Sexual behavior in Dictyna volucripes (Araneae, Dictynidae). J. Arachnol. 16: 321-330.

StatPoint Inc. 2007. STATGRAPHICS Centurion XV, version 15.2.05. Warrenton, Virginia, USA.

Uhl, G., B. Huber \& W. Rose. 1995. Male pedipalp morphology and copulatory mechanism in Pholcus phalangioides (Fuesslin, 1775) (Araneae, Pholcidae). Bull. Br. Arachnol. Soc. 10: 1-9.

Uhl, G., S. Nessler \& J. Schneider. 2007. Copulatory mechanism in a sexually cannibalistic spider with genital mutilation (Araneae: Araneidae: Argiope bruennichi). Zoology 110: 398-408. 